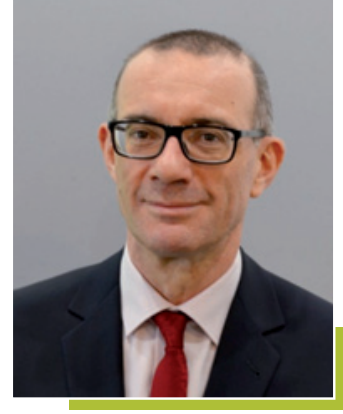

Alexandre EVIN-LECLERC

Consultant du secteur public

\title{
La mobilisation des équipes dans un projet de transformation et de digitalisation de la fonction finance
}

\author{
Mots-clés: \\ gestion publique - gestion locale - administration numérique
}

La digitalisation de la fonction finance est en marche dans les collectivités territoriales. Les exemples montrent les liens entre digitalisation et organisation, d'une part, et entre digitalisation et gestion des ressources humaines, d'autre part.

M ercredi 19 avril 2017, la deuxième rencontre des Rendez-vous de la Transformation de la Fonction Finance était consacrée à la thématique suivante : "La mobilisation des équipes dans un projet de transformation et de digitalisation de la fonction Finance ». Une démarche de digitalisation conduit en effet à repenser en profondeur les conditions et l'organisation du travail d'une collectivité. Au-delà des inquiétudes naturelles suscitées par ce type de démarche, comment mobiliser durablement les agents et en faire les acteurs d'un changement réussi?

Coordonnés par le Forum pour la Gestion des Villes et des Collectivités Territoriales, en partenariat avec la revue Gestion et Finances Publiques, les Rendez-vous de la Transformation de la Fonction Finance forment un cycle de rencontres consacrées aux évolutions majeures de la fonction finance pour le bloc local.
Accessibles en présentiel ou en Webinar, ils s'adressent aux Directeurs Généraux des Services et aux Directeurs des Finances de collectivités. Des grands témoins, issus du secteur public et du secteur privé, sont invités à partager leurs expériences sur la thématique traitée, afin de favoriser un dialogue original sur les grands enjeux financiers du bloc local.

\section{Alexandre EVIN-LECLERC Consultant secteur public}

Alexandre EVIN-LECLERC, animateur de la rencontre, a ouvert les débats en rappelant les questions soulevées par la digitalisation, sur la place et l'organisation du travail dans toutes les organisations. II rappelle que, de Ricardo à Sauvy en passant par Schumpeter, de nombreux ouvrages ont été consacrés à l'impact du progrès technique sur le mouvement de création/destruction d'emplois. Plus proche de nous, le rapport 
Mettling de septembre 2015 «Transformation numérique et vie au travail », après avoir établi un diagnostic précis et détaillé sur les impacts de la numérisation de nos cadres de travail, proposait 36 préconisations pour adapter nos organisations et accompagner les mutations sociales.

Aujourd'hui, comme les entreprises, les collectivités territoriales sont concernées par l'enjeu du digital, hier par la mise en place de la procédure SEPA' ou l'implémentation d'ERP', aujourd'hui par la dématérialisation des factures, demain par la généralisation de l'open data. Ces différentes (r)évolutions ont pour le moment été bien adoptées, mais nécessitent de mettre en place de nouvelles organisations, de nouveaux modes de faire.

C'est notamment le cas pour les directions des Finances, en mutation constante. Si elles restent garantes de la fiabilité de la chaîne comptable, elles doivent aussi contribuer à une adaptation du changement pour leur direction et l'ensemble des autres directions de la collectivité

La transformation digitale appelle ainsi à penser et définir un projet collectif et global, comportant un volet juridique, technique, métier, managérial et surtout, humain.

\section{Romain ROGUET}

Directeur Général Adjoint aux Finances de la Métropole européenne de Lille

Roman ROGUET est revenu sur l'expérience de la Métropole Européenne de Lille dans le déploiement du chantier de transformation digitale de la fonction finance.

"La dématérialisation à la Métropole européenne de Lille s'inscrit dans un projet plus global de réorganisation des fonctions support "

1. La mise en place de la dématérialisation de la fonction finance visait, outre le respect d'obligations légales, à obtenir une chaîne d'exécution financière plus efficace, avec pour conséquence directe des gains de temps et de charges, et une amélioration continue des processus.

Ces objectifs ont été atteints par une massification et une automatisation des opérations récurrentes à faible valeur ajoutée (calculs d'indexation, liquidation...), ainsi qu'une " coupe » des pertes de temps (transmission d'informations entre deux services, délais d'informations...).

La maîtrise de la chaîne financière a également permis d'obtenir une visibilité sur les dépenses et les recettes "en stock», une uniformisation des procédures, ainsi qu'une amélioration continue des processus en allant au plus simple et au plus efficace. De plus, en choisissant de résoudre en une seule fois les difficultés, plutôt que les unes après les autres, des solutions ont été mises en $œ u v r e$ par et pour toute la collectivité. Enfin, tous ces choix ont permis une montée en compétences des agents et une visibilité des expertises.

2. La digitalisation a été possible grâce à l'implication des opérationnels, un outillage performant et une information pertinente. En effet, la lisibilité de la chaîne financière s'est construite par une responsabilisation des opérationnels, une répartition des rôles plus claire entre opérationnels et financiers et enfin, une information enrichie et des processus prévisibles.

En replaçant les responsabilités là où elles doivent être, les opérationnels sont devenus responsables de leur projet et ce, grâce aux fonctions support qui les ont "équipés » : conseils, procédures administratives, système de pilotage...

"Les finances sont l'affaire de tous les agents, même des opérationnels!»

Il faut également retenir que permettre la dématérialisation, c'est adapter les procédures aux contraintes de la dématérialisation et avoir une structure suffisamment agile et maîtrisée pour dépasser les blocages techniques. Afin d'arriver à une dématérialisation de bout en bout, il a fallu, concernant la Métropole européenne de Lille, adopter une approche par processus : quels sont les besoins et comment les satisfaire?

Comme d'autres collectivités qui travaillent ou qui ont déjà mis en place la dématérialisation, il a également fallu scanner en masse les entrées, mais aussi maintenir un parcours du document $100 \%$ dématérialisé en interne.

La dématérialisation est une démarche progressive. C'est pourquoi il faut accepter de se laisser du temps: ne pas attendre que l'infrastructure soit complète pour dématérialiser et, une fois la base acquise, privilégier les «briques » à plusvalue : choix des directions à dématérialiser en priorité, choix des fonctionnalités...

Dans ce cadre, la réorganisation a permis de placer la chaîne financière sous un même commandement et de dématérialiser la totalité de la chaine commande-paiement par l'exploitation de logiciels comptables.

3. Pour mener à bien ce genre de projet, il faut qu'il soit soutenu par la hiérarchie. Concernant la Métropole européenne de Lille, I'implication du Directeur Général des Services, par le biais d'animation de réunions spécifiques avec les agents des fonctions support et un rappel régulier de la transformation digitale et organisationnelle à l'occasion des discours, réunions... a été plus qu'essentiel à la réussite du projet. 
Les DGA et directeurs ont également été régulièrement informés sur le projet. Des échanges réguliers ont été mis en place pour anticiper les difficultés et impliquer la hiérarchie.

De plus, un important travail de formation du service de la dématérialisation comptable aux Finances a été organisé (110 séances de formation, 640 agents opérationnels formés et 60 financiers) ainsi qu'une assistance de chaque groupe de directions, sur place, une fois par semaine pendant trois mois après la bascule à la dématérialisation.

Le déploiement du projet s'est également appuyé sur la Direction des Ressources Humaines. Celle-ci a participé à l'équipe projet, a permis I'organisation d'un forum de la mobilité spécifique et à procéder à des rencontres individuelles avec les agents le souhaitant (44 en tout).

4. La Métropole Européenne de Lille a également choisi d'associer les agents.

Elle a ainsi mis en place une structure de pilotage connue de tous : Chef de projet (DGA Finances), Comité de pilotage (DGS + DGA), Comité technique (1 représentant par pôle) et des groupes de travail (4-5 réunions par fonction) mêlant opérationnels et fonctionnels. Ceci a permis d'effectuer un travail sur la répartition des tâches et sur les procédures structurantes.

De plus, des conférences d'informations régulières (trois sessions en neuf mois dont deux portant spécifiquement sur la dématérialisation) ont été organisées à chaque étape du projet, spécifiques aux agents concernés mais aussi -sur une base volontaire- à tous ceux de la Métropole.

Enfin, le projet s'est déployé en toute transparence. Tous les documents ont été mis à disposition de tous les agents sur un espace collaboratif avec la possibilité de réagir et de faire part de proposition en ligne ou par mail.

5. Un an après le déploiement de la dématérialisation, un premier bilan a été présenté.

Concernant le volet « Ressources Humaines », 187 agents étaient directement concernés. II a été observé :

- Une réduction des effectifs liés aux fonctions support,

- $15 \%$ de «turnover » dans les postes comptables... mais certains cherchent à revenir,

- $20 \%$ des postes «Appui et pilotage » qui ont été ou seront réorientés « sous le manteau » par les directions opérationnelles vers d'autres besoins.

Concernant le volet technique, la Métropole a pu se rendre compte que davantage de factures étaient traitées (+ 2,8\%), qu'il y avait, en valeur, moins de stock restant en fin d'année, donc qu'un meilleur ciblage des factures à enjeux avait été fait. Cela a permis d'obtenir une clôture financière beaucoup plus juste : I'atterrissage comptable s'est fait à $20 \mathrm{M} €$ près en 2014 contre $1 \mathrm{M} €$ près en 2016 !

Notons également que la dématérialisation et centralisation de la chaine financière ont été indissociables. Les blocages de la dématérialisation ont été essentiellement le fait de pratiques "divergentes». L'ordinateur ne connaissant qu'une ou deux manières de faire, il a fallu harmoniser les procédures. Celles-ci ont par ailleurs été accueillies assez favorablement par les opérationnels. Enfin, la maîtrise de la totalité de la chaîne a permis une réactivité suffisante pour résoudre les blocages en adaptant les procédures.

Au final, la réforme a été assez bien comprise par les agents. Sur la base d'une enquête satisfaction menée auprès de 2500 agents et à laquelle 140 ont répondu, $64 \%$ connaissent les objectifs de la réorganisation et 85 \% connaissent le rôle des CSP3.

En mettant en place une stratégie d'accompagnement et de formation auprès des équipes, le projet s'est donc mis en place «facilement».

6. Certains facteurs clés de succès sont identifiables. Le soutien de la Direction Générale a été primordial. Mais il faut également souligner la confiance des équipes Finances et Commande Publique dans leurs cadres, ainsi que le maintien de relations de proximité entre ces agents et les opérationnels. Enfin, le dialogue avec les organisations syndicales, à chaque étape de décision, a permis d'éviter de créer des points de blocage et a permis d'intégrer les préoccupations concernant les conditions de travail des agents.

7. La démarche a créé des « effets secondaires» intéressants, à commencer par l'objectivisation des charges de travail, la mise en exergue de manières de faire chronophages et une implication des opérationnels sur le volet financier.

Bien évidemment, avec la dématérialisation, le métier comptable a été directement impacté. Si la standardisation et l'automatisation de certaines tâches a permis une entrée dans le métier plus simple, elles ont eu pour conséquence d'appauvrir certaines missions. II a fallu repenser le métier, l'enrichir de références techniques ou managériales, avec l'encadrement de micro-équipes de 3-4 personnes par exemple. La dématérialisation a donc permis une progression «à la carte » des missions des agents en fonction de leurs compétences ainsi qu'un repositionnement des missions des cadres.
${ }^{3}$ Contrat de sécurisation professionnelle. 
8. Les points de vigilance sont tout d'abord techniques : établir un partenariat avec la SSII ${ }^{4}$; garantir une réelle maîtrise d'ouvrage financière pour comprendre la technique et ses implications sur I'activité des agents financiers et opérationnels; s'assurer de la réactivité des éditeurs dans la résolution de «bugs »... La mise au point d'un schéma technico-fonctionnel est donc indispensable.

Les points de vigilance sont également du ressort des ressources humaines : impliquer les agents, même si on n'en sent pas toujours immédiatement la valeur ajoutée; informer régulièrement sur les évolutions des outils et des procédures; mettre en place des référents dans les services; maintenir les liens entre les financiers et les opérationnels...

9. Pour 2017, les perspectives attendues portent, tout d'abord, sur la valeur ajoutée de la dématérialisation :

- Simplifier les procédures (par exemple : définition avec le comptable d'une liste fermée de pièces justificatives de dépense, limiter le type des formules de révision...);

- Développer des cadres de référence et des aides (outils en ligne, tableur de calcul comportant l'impact des indices mis à disposition tous les agents, enrichissement des tableaux de bord des opérationnels et des cadres.

D'autres points seront travaillés comme le renforcement de la synergie marchés publics/finance, la stabilisation technique de la dématérialisation ou la révision du dispositif de support fonctionnel. En effet, avec la dématérialisation, la Métropole est passée de 200 à 900 utilisateurs du système d'information financier, dont certains sont des techniciens peu informés des questions financières ou peu familiers avec l'outil informatique. Cela nécessite de continuer une communication importante auprès de tous les agents concernés et l'organisation de cycles de formations internes Marchés et Finance. Celles-ci auront pour but de donner des connaissances de bases obligatoires pour les nouveaux arrivants et les cadres. Enfin, des cycles plus pointus pour les agents financiers, mais ouverts à tous les agents, seront proposés afin de permettre une mise à jour régulière des connaissances.

\section{Christophe ROBLIN}

\section{Consultant Interne Senior « Conduite du changement et Transformation Digitale » chez Orange}

Christophe ROBLIN a expliqué comment Orange s'est préparé et accompagne ses équipes dans une conduite de changement digital; comment
Orange a fait de l'expérience salariée la base de cette démarche.

1. À l'ère du digital, la transformation numérique peut sembler oublier, dans les entreprises, I'aspect humain. Afin de remédier à ce risque, une " ambition RH » a été pensée chez Orange dès 2010, et réécrite en 2015. Elle vise à faire d'Orange un employeur digital et humain.

Aujourd'hui, c'est la société civile qui pousse les entreprises à évoluer. Mieux encore, chaque échelon hiérarchique apporte quelque chose aux autres. Et face à la présence - historique - de plusieurs générations au sein d'une même entreprise, il fallait résoudre une équation : comment faire travailler ces différentes populations ensemble?

"Chez Orange, il n'est pas rare de voir des apprentis "éduquer" leurs collègues. Nous appliquons le principe novateur du monitoring reverse!»

Orange a ainsi choisi une stratégie axée sur l'expérience salariée afin de comprendre ce que l'employé va vivre. Cette expérience repose sur trois points : les compétences, l'agilité collective et l'engagement individuel.

2. Afin de mener à bien sa transformation digitale, Orange a créé une Digital Academy, visant à former les salariés au numérique. Cela passe par I'utilisation des réseaux sociaux, la création d'un réseau social interne ou encore, des visas métiers, pour tous ceux qui veulent se former sur les questions du numérique. Les méthodes de management ont été revues et adaptées. Des recrutements internes de salariés, connaissant bien l'entreprise, ont été réalisés afin de mettre en place une méthode de type " change management ». Des groupes, non pas de salariés, mais "d'intelligences», ont ainsi été créés pour participer au changement.

Il faut un fort soutien managérial pour réussir une transformation digitale. Ce soutien doit venir aussi bien de la Direction Générale, que des managers. En effet, la transformation digitale inclut presque irrémédiablement une perte de pouvoir de la hiérarchie. C'est pourquoi il faut comprendre que le rôle d'un manager tend à évoluer. Aujourd'hui, le manager doit guider l'intelligence collective, tout en acceptant que d'autres membres de l'équipe prennent parfois le leadership sur des questions particulières qui peuvent lui échapper.

Afin d'amener progressivement la transformation digitale au sein d'Orange, il a également fallu faire comprendre que le changement serait le même pour tous. Et surtout, afin de dissiper les doutes ou angoisses des salariés réfractaires, demander «Si on ne change rien, qu'est-ce qui se passe?». 
C'est en posant ce genre de question que la révolution numérique a pu se mettre en place.

Dès septembre 2014, une équipe dévolue à la transformation digitale a été créée, en accord avec la Direction de l'Innovation. Celle-ci a permis d'accompagner les projets et l'expérimentation au sein de l'entreprise mais également d'éviter l'opposition entre les différentes directions.

Afin d'amener la transformation, il a également été décidé de mettre à disposition toutes les informations concernant les conduites de projet. Chacun peut donc suivre les évolutions et propositions. Orange a ainsi joué la transparence auprès des équipes.

Pour accompagner davantage les salariés, un système de formation a été mis en place: Orange Learning. Ce système propose des formations en présentiel, mais aussi en différé $\left(M O O C^{5}\right.$ par exemple). De cette façon, chacun y trouve son compte et monte en compétence à son rythme.

3. Une stratégie a dû être pensée pour mener à bien le projet de digitalisation. Celle-ci a commencé par une analyse du besoin et une réflexion organisationnelle, préalables à la préparation de la mise en œuvre par phases.

L'analyse du besoin s'est construite par une audition individuelle des 10 DGAS, de l'ensemble des directeurs, des SG et des chefs de GFAJ et une analyse de ces auditions.

Suite à cela, la réflexion a pu se construire par un travail sur les conséquences organisationnelles du projet et une définition du rôle des cellules de pilotage et d'appui (CPA) et de leurs responsables.

Il est essentiel d'identifier les acteurs du changement. Chez Orange, par exemple, des réseaux de facilitateurs ont été créés afin de communiquer facilement sur les changements.

La préparation de la mise en œuvre s'est ensuite faite par l'adaptation des process et des outils, ainsi que celle des organigrammes, en adéquation avec l'organisation cible. Tout ceci a permis au final une mise en œuvre progressive selon les thématiques et un accompagnement.

"Il est important de célébrer les succès, mais également les échecs pour apprendre de nos erreurs ».

4. Il est très important d'avoir au préalable définis les critères cibles: "Nous aurons réussi si/quand... ". Cela permet aux collaborateurs $d^{\prime}$ avoir une vision plus claire sur les changements à venir. Les investissements/gains/bénéfices sont parfois difficiles à identifier, mais au fur et à mesure des étapes, ils apparaissent d'eux-mêmes et sont compris.

De plus, à chaque étape, il est essentiel de «sonder » les collaborateurs. Ceci permet, d'une part, de leur montrer ce que l'on aimerait obtenir pour les équipes et, d'autre part, qu'ils expriment ce qu'ils souhaitent. Ces échanges permettent également d'adapter les discours pour accompagner les collaborateurs et mener une conduite du changement. On en revient donc à une notion essentielle : la communication.

En 2010, Orange a mis en place un baromètre social, qui interroge les collaborateurs par échantillonnage. Celui-ci touche toutes les composantes de l'entreprise. C'est aussi un élément de discussion avec les organisations syndicales, qui permet de mesurer ce qui fonctionne ou ce qui doit être corrigé. Aujourd'hui, avec la transformation digitale, ce baromètre évolue. C'est pourquoi Orange propose à ses collaborateurs, par le biais d'une application interne, de juger et dire s'ils se sentent bien dans la conduite du changement. De plus, en proposant ce type d'outil, les « réfractaires » au changement intègrent petit à petit de nouveaux outils à leurs missions.

Le changement se fait pas à pas, tant dans le public que le privé. Mais en accompagnant les équipes, et les formant et en les écoutant, tout changement peut être une réussite.

\section{Conclusion}

Des échanges et débats qui ont eu lieu, 3 facteurs clés de succès du déploiement d'une démarche digitale et d'une mobilisation des équipes peuvent être identifiés :

1. Tout projet de déploiement d'un projet de digitalisation doit être ambitieux et fixer un cap à moyen/long terme : mais il doit être également pragmatique, concret, se traduire rapidement par des gains visibles, mêmes modestes, par les équipes

2. Il faut choisir ses combats, savoir prioriser : la maturité numérique est un processus long; la " politique des petits pas » est donc préférable au " grand soir»!

3. Accepter ses erreurs, savoir se former à tout âge, se remettre en question sont quelques-unes des attitudes qui permettent à tous d'être agiles et contributifs à la réussite du projet.

Bref, pas de réforme réussie sans mobilisation du capital humain! 